\title{
Visualisation to Aid Biodiversity Studies through Accurate Taxonomic Reconciliation
}

\author{
Martin Graham ${ }^{1}$, Paul Craig ${ }^{1}$, and Jessie Kennedy ${ }^{1}$ \\ ${ }^{1}$ Centre for Information and Software Systems, Napier University, 10 Colinton Road, \\ Edinburgh, EH10 5DT, UK \\ \{m.graham, p.craig, j.kennedy\}@napier.ac.uk
}

\begin{abstract}
All aspects of organismal biology rely on the accurate identification of specimens described and observed. This is particularly important for ecological surveys of biodiversity, where organisms must be identified and labelled, both for the purposes of the original research, but also to allow reinterpretation or reuse of collected data by subsequent research projects. Yet it is now clear that biological names in isolation are unsuitable as unique identifiers for organisms. Much modern research in ecology is based on the integration (and re-use) of multiple datasets which are inherently complex, reflecting any of the many spatial and temporal environmental factors and organismal interactions that contribute to a given ecosystem. We describe visualization tools that aid in the process of building concept relations between related classifications and then in understanding the effects of using these relations to match across sets of classifications.
\end{abstract}

Keywords: Biodiversity, taxonomy, concepts, concept relationships, visualisation

\section{Introduction}

Consider a typical research scenario: a scientist is interested in analyzing the spread of invasive species in a certain region [1]. They are aware of pertinent results in the literature and have additional distribution records in their personal database, along with access to other potentially relevant datasets on-line. The researcher needs to be able to discover candidate datasets and be able to merge relevant and compatible information from these varied datasets. Simplistically, datasets might be retrieved and integrated on the basis of country and species name; however, just as country names and boundaries change over time, so do the definitions attached to species names. Unfortunately sufficiently sophisticated on-line taxonomic resources and tools to aid the biologist are not currently available to allow them to address these problems in legacy data sets or to adequately annotate new data sets. An essential step for reusability and longevity of these data is the documentation of the contents of such datasets, but effective documentation depends on the implementation of adequate 
practices and information technologies $[2 ; 3]$ as well as adherence to defined data standards.

Ecological data may refer to organisms in a variety of ways: by their common name, by some internal code, or by scientific name. Although appearing to be less ambiguous than local common names, scientific names are also unstable and change in meaning over time and between authorities. The name used in one ecological study will reflect the classification context used by the authors at that time; datasets produced at different times or by different workers in different geographical locations might reference competing, if not conflicting, taxonomic standards [4; 5]. Hence the inconsistent meaning of taxonomic labels used to identify species or higher-level taxonomic groups necessitates semantic integration of the data for ecological analyses. Furthermore, if the context or source for a recorded name is not captured as part of the documentation of the dataset, it can be impossible for subsequent workers to accurately resolve relationships among taxa identified simply by name.

The exact meaning of taxonomic names can change over time due to the lumping, splitting, or redefinition of lineages as taxonomists revise their classifications [6], and might also vary significantly between contemporary treatments owing to differences in interpretation, (e.g. morphological versus genetic criteria) or circumscription (i.e. the limits or extent of a given taxon). The valid scientific names applied to taxa (i.e., species or other groups) in a given taxonomic classification are mechanistically determined according to codified rules of nomenclature via the method of typification and the principle of priority [7]. Because taxonomic opinion and classifications evolve, the names that are properly applied to revised taxonomic concepts may be identical to those used to refer to earlier and possibly quite different circumscriptions of taxonomic entities. To share a name, two taxon circumscriptions need only include the same type specimen. A further consequence of the application of the nomenclatural codes to the results of taxonomic revision is that different names may be used according to different taxonomic perspectives; yet refer to entities that appear indistinguishable. As a consequence of these problems of synonymy and homonymy it is impossible to integrate multiple datasets with any certainty of accuracy simply by matching the names of taxa they contain. Thus, taxonomic names are a significant and pervasive source of ambiguity when dealing with biodiversity data of mixed provenance [8].

An example serves to illustrate the problem. Alternative taxonomies arise with the discovery of new specimens and species, more information about shared traits and newly inferred phylogenetic relationships, and even new analytical tools [9]. Consider the example of gorilla taxonomy [10] (partially) in Fig. 1. Gorillas were first described and classified by Reverend Savage in 1847, based on a population found in West-Central Africa. He considered them similar to chimpanzees (named Troglodytes niger in 1812 by E. Geoffroy St. Hilaire) and grouped them into the same Genus calling them Troglodytes gorilla. However in 1816, Oken realized that the generic name Troglodytes had already been used in 1806 by Vieillot to name the bird wren, therefore the generic name for chimpanzees was changed to Pan, (strictly speaking Savage \& Wyman should've named gorillas Pan gorilla in 1847). However, in 1852 I. Geoffroy St. Hilaire re-classified the gorillas, separating them from chimpanzees and renaming them Gorilla gorilla, the first use of the name for gorillas commonly used today. In 1902, they were found in East-Central Africa and in 1903 Matschie 


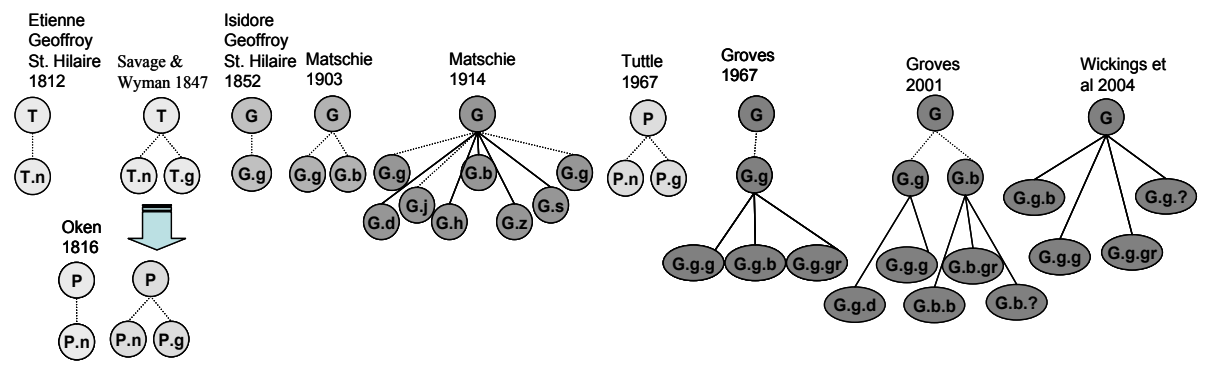

Fig. 1. Summary Gorilla Classifications showing genus, species and sub-species as classified by some of the primate taxonomists since gorillas were first discovered in 1847 through to 2004.

reclassified them and defined a new species of gorilla called G. berengei. Matschie continued his splitting of gorillas, resulting in several species including G. graneri from the Congo in 1914 and many others (G. diehli, G. jacobi, G. schwarzi, G. hansmeyeri and G. zenkeri). Other described species and sub-species not shown.

In 1967 Tuttle claimed that gorillas were related to chimps and put them back in the genus Pan while Groves claimed that there was only one species of gorilla, $G$. gorilla of which there were 3 sub-species: G. g. gorilla, G. g. graueri and G.g.beringei but by 2001 with more recent evidence Groves had reclassified Gorilla into two species (currently agreed by most experts in the field) and 5 sub-species: $G$. gorilla, G. g. gorilla, G. g. diehli and G. beringei, G. b. beringei, G. b. graueri and an un-named subspecies of $G$. $b$. However recent DNA analysis [11] is suggesting that four distinct evolutionary specific units of gorilla exist although it is not clear if these are species or sub-species. In addition to taxonomic treatments, if we consider the popular field guides for example Mammal Species of the World, in the 1993 revision it presented gorillas as one species G. gorilla with 3 subspecies, similar to Groves 1967. However in the 2005 edition they are adopting the Groves 2001 classification with 2 species and 4 subspecies. Apes of the World on the other hand adopt Tuttle's definition of gorilla and recognize 1 species $P$. gorilla.

It should now be clear that when we use the taxonomic name G. gorilla there may indeed be many different definitions of this name; what we refer to as taxon concepts (the taxon name as defined by a particular taxonomist in a particular classification). Just at species level in the gorilla example above we have 10 different taxon names that are used with varying meanings across 8 different classifications, (i.e. we have 18 different taxon concepts which have been described by the authors). However the current "accepted" treatment used varies between individuals and institutions, and therefore biologists undertaking analysis of data which may have been collected according to different field guides could end up with misleading results in their analysis unless they were knew if it was about G. gorilla Groves 1967, as compared to $G$ gorilla Groves 2001 or $P$. gorilla. In databases and in the literature however authors are frequently vague about what taxon concept they are referring to, and simply cite a name such as G. gorilla thinking this makes it clear as to what they meant (and for something as well known as gorillas who wouldn't?). This can also have serious repercussions, for example, in conservation if the red list (and associated statute) cites G. gorilla (according to Groves 1967 but without explicitly specifying so) as endangered and illegal to trade, then a poacher might be able to legally argue 
that trading in G. berengei (according to Matschie or Groves 2001) is legal as it's a different species.

If ecological information is to be useful for future analyses, it should document against what treatment the original authors of the data identified the organisms, and long-term strategies to manage the legacy of ecological data must accept and accommodate this constraint [12]. Minimally, a reference to the source publication (e.g. field guide) used in making taxonomic identifications should be included, to indicate the taxonomic concept being identified. New or annotated data should record 'taxon concepts' which capture the differences in names shown by the case studies in Fig. 1, rather than simple scientific names. The adopted convention for referring to taxonomic concepts is to cite the name sec. author [4] where "sec." stands for the Latin secundum, meaning "according to" a particular (team of) author(s). It provides (inter alia) a solution to the problems discussed. We can now refer to Gorilla gorilla Savage \& Wyman sec. Groves (2001), for example and use this as a reference for the underlying definition. To this end, an abstract model, the Taxonomic Concept Schema (TCS) for representing taxonomic concepts and their relationships has been developed [13] and ratified by the International Biodiversity Standards (TDWG) as one of their standards. This standard will facilitate merging ecological datasets collected at different times and places by workers following varied taxonomic standards.

Research has been undertaken to determine the stability of names relative to taxonomic concepts [14] using Koperski et al's moss data set as example data [15]. It was revealed that only $13 \%$ of Koperski et al's taxa declared congruent relationships to concepts with the same name in different classifications and made no other relationships. Another $22 \%$ of the taxa had congruent relationships only, but this time to homotypic synonyms, a further $20 \%$ had doubtful stability and $45 \%$ had various incongruent relationship types, indicating instability between taxonomies.

Recent work [16] reports similar issues in weevil data, reinforcing the message that researchers can only depend on a minority of names in one classification actually meaning the same concept in another, and this ratio tends to diminish as time passes between classifications. Thus with names unsuitable as cross-taxonomy identifiers, concepts and concept relationships come to the forefront of linking and matching between taxonomies. What are needed now are tools to enable taxonomists to accurately mark up their data with concepts and relate new revisions to existing legacy classifications so vital for long term biodiversity studies, and for other specialists to be able to effectively use this data.

\section{Visualisation}

One approach for exploring and generating the structures formed through the concepts and their relationships is Information Visualisation (IV) [17] - the graphical display of and interaction with complex data sets. Essentially we have a set of multiple hierarchies - the classifications we are attempting to reconcile - along with a set of links that map between those hierarchies - which are the concept relations. To this end we have developed two complementary visualizations for our data; the first, TaxVis, allows users to explore relationships between multiple classifications, and the 
second, Concept Relation Editor, allows concept relationships to be placed between pairs of classifications.

\subsection{TCS Relationship Data}

Both visualisations take TCS data in the form of an XML file that contains descriptions of publications, names and relationships to generate a multiple tree model with the relations and names acting as links between the publications (the classifications). The relationships themselves are defined in the data set, and are assumed to be the work of taxonomists whose names are associated with those relations. Inferring further TCS relations from existing relationships is a subject that is approached formally in [18] and discussed informally in [19]. In short we do not infer relationships in our visualizations beyond enforcing reciprocality, i.e. if A includes B, we make a relationship that $\mathrm{B}$ is included in $\mathrm{A}$, similarly if $\mathrm{C}$ overlaps $\mathrm{D}$, then $\mathrm{D}$ must also overlap C.

Generally inferring relationships is problematic as it produces a relationship which is only as strong as its weakest link i.e. if $\mathrm{A}$ is congruent to $\mathrm{B}$ which overlaps $\mathrm{C}$ we can only conclude A has an overlap with $\mathrm{C}$. Further, if $\mathrm{A}$ is included in $\mathrm{B}$ which overlaps C, we cannot even infer the overlap, as the part of B which overlaps C may not be the part which includes A. Add on top of that the difficulty of chaining relationships defined by different authors who may well have different opinions and the trustworthiness of inferred relations quickly deteriorates. Conceivably one workable scenario is to chain congruent relationships by the same author, but so far we leave further relation inferencing to the user to perform in the visualization.

\subsection{TaxVis - A Visualisation for Exploring Multiple Classifications}

We have developed a visual taxonomy explorer which allows matches between classifications to be explored through concept relationships [19]. The visualization itself consists of a number of types of different co-ordinated views applicable to a classification data set, known as a multiform visualization [20], though in the following discussion we will concern ourselves mainly with only one of the views in particular - the multiple tree view [21]. In this view, classifications are displayed as individual top-down hierarchies. Selections that are made in one hierarchy are reflected in the other hierarchies giving a measure of overlap and distribution between related classifications.

One of the most revealing tasks that can be performed in the application is to compare the differences in matches made through naïve name matching to those made through following concept relationships. Using a TCS version of Koperski et al's [15] moss concept data as input to the visualization we can show the numerous differences generated by the two approaches for a sample genus, Eurhynchium. Matching between just three of the classifications in the set, Koperski's recent revision and Smith's 1980 and Mönkemeyer's 1927 classifications, demonstrates the type of differences that can be observed. 
Firstly, when matching strictly by name, it becomes obvious even before visualization that there will be no inter-genus relationships. The species $X . a$ in one classification cannot be matched to anything other than $X . a$ in another classification. At higher levels in a classification families, classes, and even genera themselves could freely be repositioned in higher taxa without necessarily requiring re-naming - which in itself can cause problems with homonymy as people assume the same name means the same thing in different classifications. This is not so with species, a species moved between two genera must take on the name of its parent genus as the first part of its binomial name. Thus, when matching Eurhynchium across the two classifications, from Koperski back to Mönkemeyer all that happens is that the Eurhynchium genus representation is highlighted in both classifications, as seen in Fig. 2. Differences in species naming and authoring means none of the Eurhynchium species match by name. Smith's 1980 classification fares better, being closer in time to Koperksi et al's revision, with several name matches highlighted, though there are still several nodes that remain unaffected, indicating no name match.

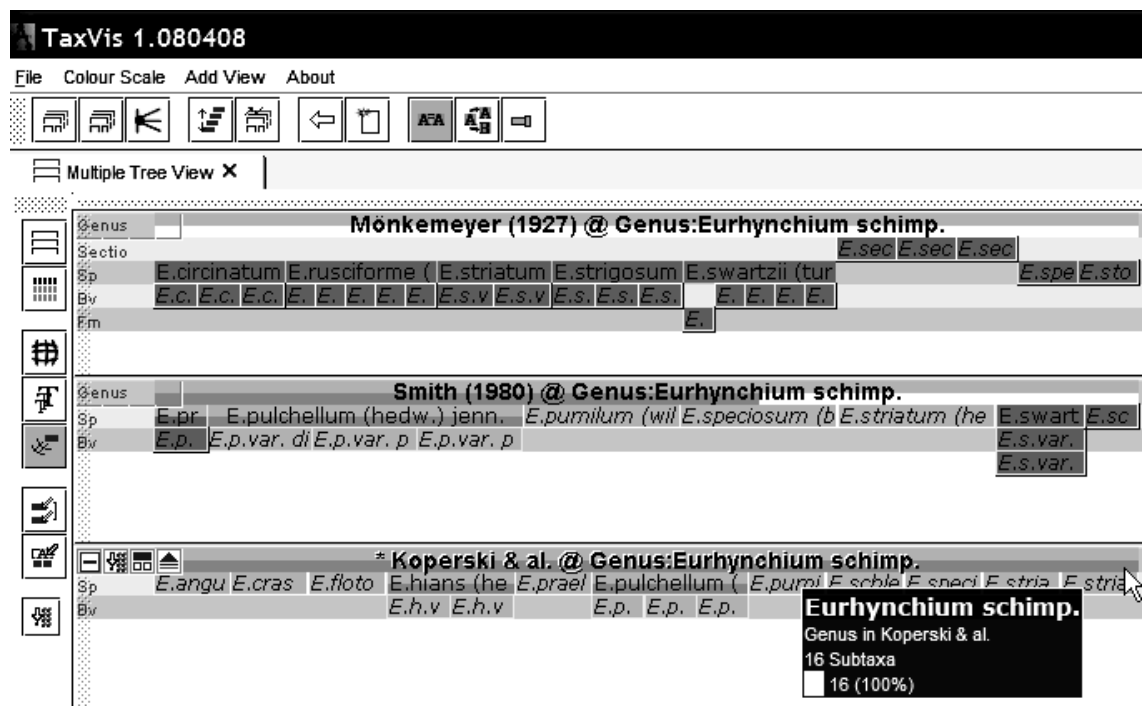

Fig. 2. Eurhynchium taxa selected by name in Koperski have little impact on Mönkemeyer.

By contrast when matching by concept relations a completely different picture emerges, as seen in Fig. 3. Koperski et al's Eurhynchium has been selected and the visualization set to highlight matches by concept relationship. This reveals relationships to four different genera in Mönkemeyer, including its interpretation of Eurhynchium, so according to Koperski et al, their definition of the genus is made from parts of those four different genera, indicating that integrating data collected under these two classifications could be troublesome. Smith's classification contains three genera that overlap with Koperski et al's treatment of Eurhynchium. However, exploration of Eurhynchium's species and varieties in Koperski et al shows that at a lower level many of the relationships are congruencies, albeit between concepts with different names. For example, in Fig. 3, the mouse is currently hovering over Eurhynchium striatalum in Koperski et al, and links and a tool tip reveal this name in 
Koperski is considered conceptually equivalent to a species of Isothecium in Mönkemeyer and Smith's classifications, though a differently named species in each.

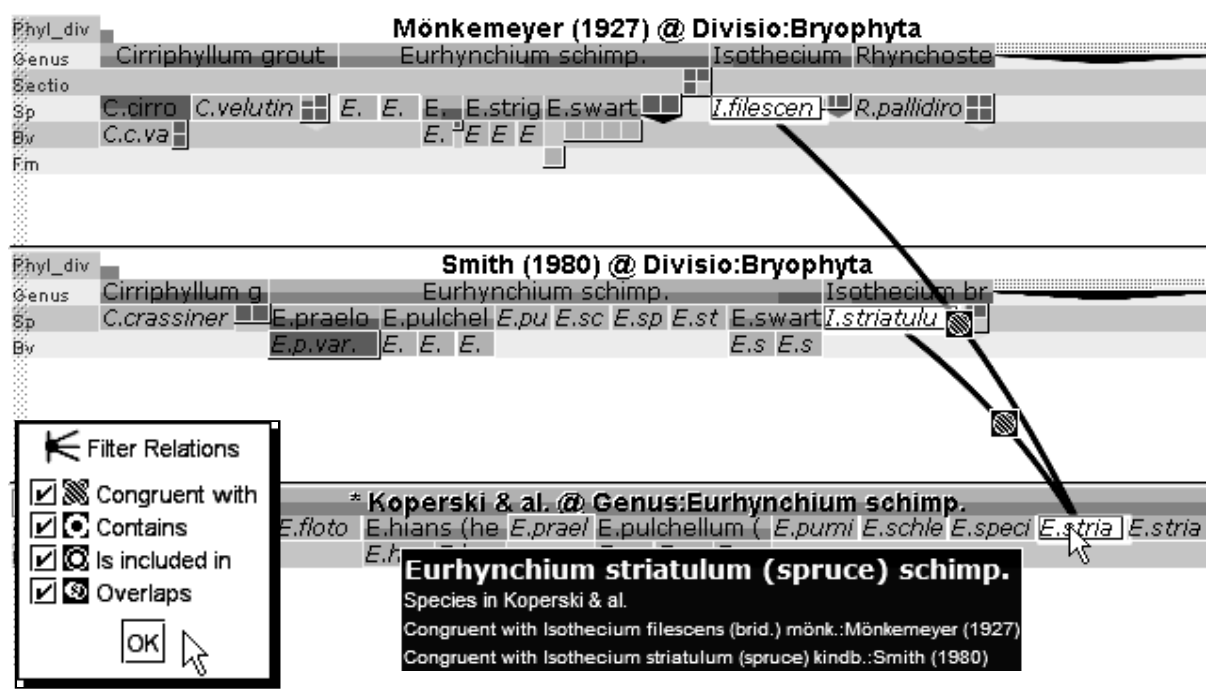

Fig. 3. The result of matching Koperski et al's Eurhynchium genus and its contents by concept relations reveal it is split between four genera in Mönkemeyer and three in Smith. A tool tip currently displays relationships for the striatulum species and shows matches to species in the Isothecium genus in the other classifications. A relation filter control is included as a key.

The moss set is simplified by the fact the relationships are all defined from one classification (Koperski et al) to the other classifications in the data set, and are all authored by the same people (Koperski et al). Other data sets may not assume these constraints, indeed we have a Ranunculus data set where relations are concentrated around two classifications and the relation set includes relations defined by two differing authors. In these circumstances it can be useful to filter out relations defined by one or more authors as taken as a whole the relationship set can contain apparently contradictory findings as seen in Fig. 4, or as stated in the previous section can lead to erroneous conclusions if relations defined by more than one author are chained together to deduce other relations.

Thus, we can visually match and explore the relationships between a set of classifications to reveal patterns that would be non-existent using naïve name matching. Using this tool, ecologists with names collected under more than one classification can match such data to be consistently based on just one of the referenced classifications. Another alternative is to use a third-party classification as seen in Fig. 3 above, where data collected and named using Mönkemeyer and Smith's classifications can be integrated under Koperski et al's classification, the example above showing Isothecium filescens in Mönkemeyer and Isothecium striatulum in Smith to be conceptually equivalent. Obviously on a singular basis, finding such matches could easily be done by just processing the relevant TCS and XML, but the visualisation allows users to view the stability of whole classifications by concept relationships. 


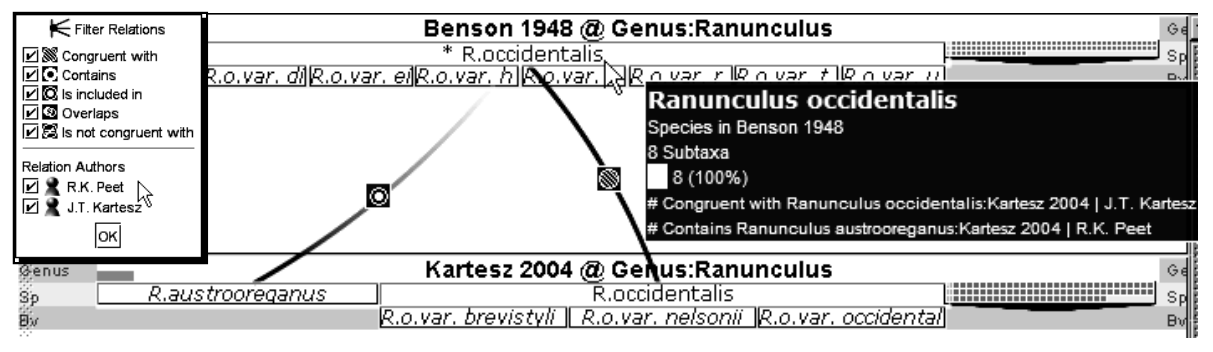

Fig. 4. Relations of R.occidentalis between Benson and Kartesz indicate an apparently contradictory situation - congruent with another species but containing another in the same classification. This is due to the relations being authored by two experts who have differing opinions. The authors and the types of relation can be filtered in or out through a pop-up menu (seen in the top left of the figure).

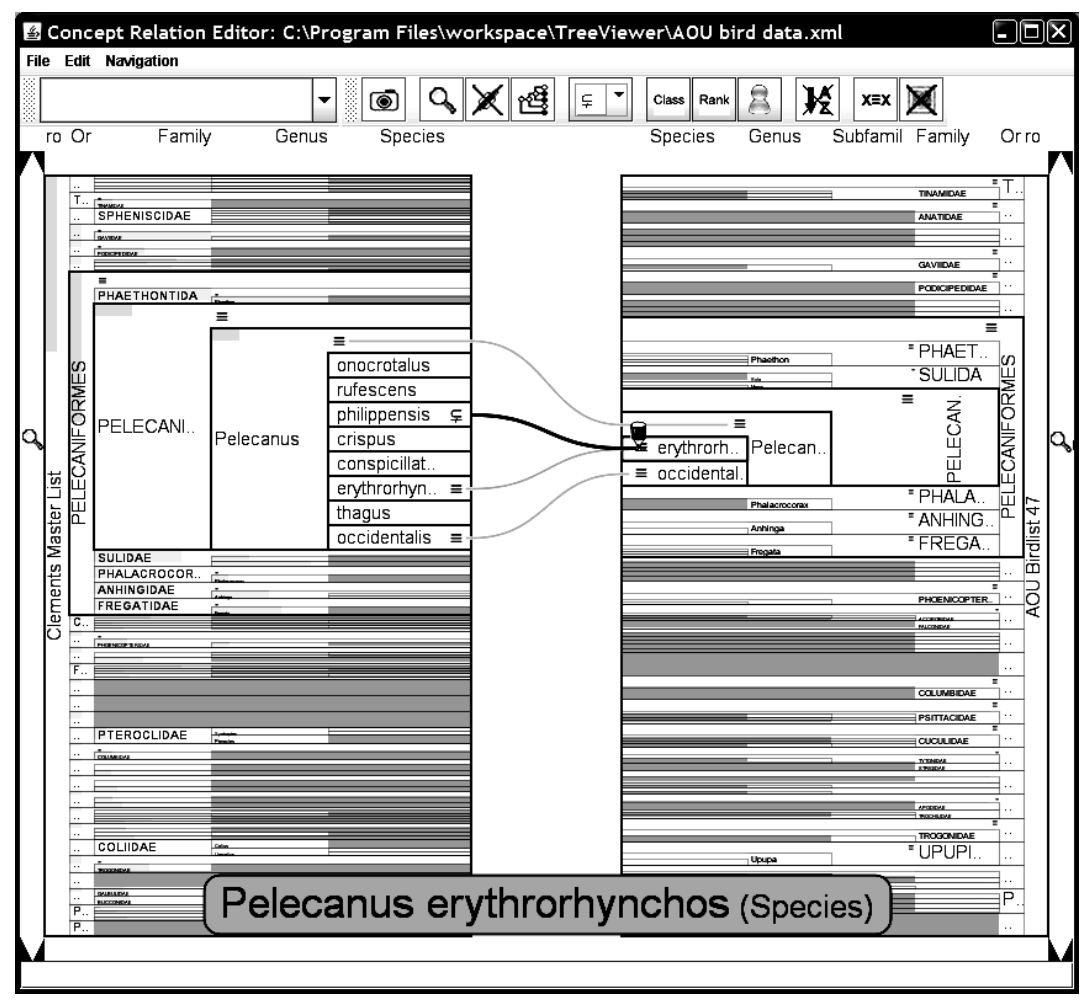

Fig. 5. The Concept Relationship Editor. Relationships are made by dragging links between taxa in opposite-facing classifications. 


\subsection{The Concept Relationship Editor}

The concept-based matching used in TaxVis is of course only as reliable as the quality of the relationship data used. In some cases there may be errors or contradictions, or just no relationship data defined at all A second visualization tool, the Concept Relation Editor (CRE) [22], was prototyped to allow taxonomists to intuitively add and edit their own relations between classifications. This could either be done for a classification the user has authored to relate it back to previous classifications, or as a third-party author inserting their own relationships between two pre-existing classifications.

In the visualization, as shown in Fig. 5, a pair of classifications is selected from the current data set and displayed facing each other on different sides of the application window. A focus +context effect [23] is applied to the trees such that selected taxa receive more space than unselected taxa, giving them room to display relationship information or for rendering child taxa. This effect can be replaced with a more traditional scroll and pan technique for large lists if the user so wishes.

Making relationships is simply a question of setting the author name and the type of relationship through the toolbar menu at the top of the display, and then dragging with the mouse a path between the taxa that are to be related. Links for selected portions of the classifications are displayed, with icons to represent the type of relationship and their permanency.

In this way taxonomists can link new classifications to legacy data, with the results being stored back in the TCS XML data for other specialists to examine

\section{Usability Study}

An informal user study was performed at the TDWG (Taxonomic Databases Working Group) conference on the two visualizations to assess their usability. Users were asked to complete sample tasks for either or both visualizations, depending on their availability and interest. They were also asked to provide comments on any problems they encountered as they proceeded, and also to give their general impression of the visualization and any further enhancements they could think of for the tools. These comments were recorded on MiniDisc audio equipment for later analysis. This "thinkaloud" [24] testing follows Nielsen's [25] prescription for using relatively small numbers of users, but making sure that they are representative users as opposed to a random sample off the street. In this case as attendees at the TDWG conference we could be sure our users were interested in working with taxonomic data.

Five volunteers tried the TaxVis visualization, attempting a scenario task based on Koperski et al's moss data set. Analysis of notes and audio recordings taken during the sessions led to a list of 17 observations and user suggestions. Most of the user suggestions and issues were based on taxonomic issues such as the incorrect capitalization of author names, drawing from their expertise in the domain, whilst the observations were mostly based on interface problems they encountered, such as being tricked by misleading colouring into attempting to select non-existent concepts. 
Table 1. Most common issues found in testing of the Concept Relation Editor

\begin{tabular}{|c|c|c|}
\hline Observation & Type & $\begin{array}{l}\text { No. of } \\
\text { users }\end{array}$ \\
\hline 1. Brushing on nodes not showing relationship lines & Observation & $* * * * *$ \\
\hline $\begin{array}{l}\text { 2. Direction of contains and contained relationships unclear } \\
\text { (one user did notice a set symbol was grey at one end of a } \\
\text { link and black at the other but didn't know what this } \\
\text { meant) }\end{array}$ & Observation & $* * * *$ \\
\hline $\begin{array}{l}\text { 3. User not equating 'taxonomies' with 'classifications', error } \\
\text { seen in labelling, ignoring button and exploring menu } \\
\text { options instead (see 35) }\end{array}$ & $\begin{array}{l}\text { Observation } \\
\text { (use user's } \\
\text { language) }\end{array}$ & $* * *$ \\
\hline $\begin{array}{l}\text { 4. Headers (name of classification) wanted on } \\
\text { taxonomy/classification selection panel }- \text { and on main } \\
\text { display (as well as / replacing the labels at the far left/right } \\
\text { of the screen) }\end{array}$ & $\begin{array}{l}\text { User } \\
\text { suggestion }\end{array}$ & $* * *$ \\
\hline $\begin{array}{l}\text { 5. User doesn't use scrollbar in lens mode, just moves mouse } \\
\text { up and down the classification and looks at the large } \\
\text { tooltip at the top of the screen }\end{array}$ & Observation & $* * *$ \\
\hline $\begin{array}{l}\text { 6. Deduced congruent relationships cannot be confirmed nor } \\
\text { deleted }\end{array}$ & Observation & $* * *$ \\
\hline $\begin{array}{l}\text { 7. Drop down list }- \text { selecting with mouse from list didn't work } \\
\text { - had to press return on the keyboard }\end{array}$ & Observation & $* * *$ \\
\hline $\begin{array}{l}\text { 8. To make a relation requires dragging to the name text rather } \\
\text { than the bounding box of the node (i.e. doesn't work when } \\
\text { released over the set symbols) }\end{array}$ & Observation & $* * *$ \\
\hline 9. User looked to open new taxonomies under 'File', not 'Edit' & Observation & $* * *$ \\
\hline
\end{tabular}

For the Concept Relation Editor we had 8 volunteers use the visualization and attempt to complete a pair of tasks based on Ranunculus and Bird data sets. Again, notes and audio recordings were taken during the sessions, leading to 42 observations and user suggestions, many of which occurred with multiple users. The observations ranged from purely interface issues such as a drop-down list initializing with an empty selection, making users unsure as to its function, to issues that required the domain expertise of taxonomists and taxonomic data managers to be flushed out. Amongst these were terminology usage (classifications versus taxonomies), default ordering of nodes (many classifications have what is known as a taxonomic ordering, which is not the default alphabetical ordering typically used) and the automatic downloading of pictures from the web to represent taxa (their accuracy was questioned). A summary of the issues that were observed or commented on by three or more users is given in the table below. For brevity we exclude the other 33 issues that were picked up by only 1 or 2 of the participants.

The discrepancy in the number of issues found between the two visualizations can be attributed mainly to the fact that the CRE tool had not been through any previous rounds of similar testing, unlike TaxVis, which as a result had many of its more 
glaring interface issues discovered and dealt with in previous incarnations. The unequal number of volunteers ( 8 for CRE compared to 5 for TaxVis) may also have contributed but as they are different interfaces we can't really compare like with like here. What we did notice in the CRE testing was the classic pattern of each additional user finding a smaller and smaller set of unique issues in an interface, but with reproduced issues reinforcing the findings of previous users. In any case, as pointed out by [26], what matters is not so much the number of issues found or users employed, but whether the findings are acted upon. In this case both TaxVis and CRE underwent significant re-engineering to address as far as possible the problems found.

\section{Summary}

We have described how a pair of related visualizations, TaxVis and the Concept Relation Editor, can be used to explore, follow and construct concept relationships across a data set of multiple classifications.

Visualising such operations offers advantages over a purely textual results service and text-based data entry approach. The most obvious being that relationships, both in their creation and in later exploration, can be viewed in the context of other relationships and concepts, and assessed in that light. This is important as conceptbased matching is inherently more complex than naïve one-to-one name matching. In TaxVis, it is possible to select and follow relationships across multiple classifications and to use the related concepts found as the starting point for further queries. In the Concept Relation Editor, users can construct their own relationship sets and observe the gradual building-up of their efforts.

Acknowledgments. Thanks to Robert Peet and Xianhua Liu for determining and encoding the concept relationships in the Ranunculus data set. Also thanks to the volunteers at the TDWG 2007 conference who gave their time and expertise to our user testing.

\section{References}

1. Higgins, S.I., Richardson, D.M., Cowling, R.M., Trinder-Smith, T.H.: Predicting the landscape-scale distribution of alien plants and their threat to plant diversity. Conversation Ecology. 13 (1999) 303-313

2. Michener, W.K., Brunt, J.W., eds. Ecological Data: Design, Management, and Processing. Blackwell Science, Oxford, 2000.

3. Foster, I., Kesselman, C., eds. The GRID 2: blueprint for a new computing infrastructure, 2nd ed. Elsevier Series in Grid Computing. Elsevier, Amsterdam, 2004.

4. Berendsohn, W.G.: The concept of "potential taxa" in databases. Taxon. 44 (1995) 207-212

5. Pullan, M.R., Watson, M.F., Kennedy, J.B., Raguenaud, C., Hyam, R.: The Prometheus Taxonomic Model. Taxon. 49 (2000) 55-75

6. Peterson, A.T., Navarro-Sigüenza, A.G.: Alternate species concepts as bases for determining priority conservation areas. Conservation Biology. 13 (1999) 427-431

7. Minelli, A.: The status of taxonomic literature. Trends in Ecology \& Evolution. 18 (2003) 
$75-76$

8. Kennedy, J.B., Kukla, R., Paterson, T.: Scientific names are ambiguous as identifiers for biological taxa: their context and definition are required for accurate data integration. 2nd International Workshop on Data Integration in the Life Sciences Ludäscher, B., Raschid, L. (eds.). LNCS, Vol 3615. Springer-Verlag Berlin Heidelberg, (2005) 80-95

9. Schuh, R.T.: Biological Systematics: Principles and Applications. Cornell University Press, Ithica, NY (2000)

10.Groves, C.P.: A history of gorilla taxonomy. In: Taylor, A.B., Goldsmith, M.L. (eds.) Gorilla biology. Cambridge University Press, Cambridge (2003) 15-34

11.Wickings, E.J., Clifford, S.L., Anthony, N.M., Jeffery, K., Johnson-Bawe, M., Abernethy, K.A., Bruford, M.W.: Gorilla mtDNA - Sequences Unravelled and Secrets Revealed. Gorilla Journal. 29 (2004)

12.Kennedy, J.: Exploiting Diverse Sources of Scientific Data. Final Theme report, e-Science Institute (2007)

13.Kennedy, J., Bowers, S., Jones, M., Madin, J., Peet, R., Pennington, D., Schildhauer, M., Stewart, A., http://www.tdwg.org/fileadmin/2007meeting/slides/Kennedy_DataIntegration IssuesSeek abs231.ppt

14.Berendsohn, W.G., Geoffroy, M.: Networking taxonomic concepts - uniting without "unitary-ism". In: Curry, G., Humphries, C. (eds.) Biodiversity Databases - Techniques, Politics, and Applications. CRC Taylor \& Francis (2007) 13-22

15.Koperski, M., Sauer, M., Braun, W., Gradstein, S.R.: Referenzliste der Moose Deutschlands. LV Druck im Landwirtschaftsverlag GmbH, Münster-Hiltrup (2000)

16.Franz, N., Peet, R., Weakley, A.: On the use of taxonomic concepts in support of biodiversity research and taxonomy. In: Wheeler, Q.D. (ed.) The New Taxonomy, Systematics Association Special Volume Series 74. Taylor \& Francis, Boca Raton, FL. (2008) 61-85

17.Card, S.K., Mackinlay, J.D., Shneiderman, B., eds. Readings in Information Visualization: Using Vision to Think, 1st ed. The Morgan Kaufmann Series in Interactive Technologies. Morgan Kaufmann, San Francisco, 1999.

18.Thau, D., Ludäscher, B.: Reasoning about Taxonomies in First-Order Logic. Ecological Informatics. 2 (2007) 195-209

19.Graham, M., Kennedy, J.: Visual exploration of alternative taxonomies through concepts. Ecological Informatics. 2 (2007) 248-261

20.Roberts, J.C.: Multiple-View and Multiform Visualization. Visual Data Exploration and Analysis VII. Erbacher, R., Pang, A., Wittenbrink, C., Roberts, J. (eds.). SPIE Vol 3960. SPIE Press Bellingham WA (2000) 176-185

21.Graham, M., Kennedy, J.: Combining linking \& focusing techniques for a multiple hierarchy visualisation. IEEE Conference on Information Visualization. Banissi, E., Khosrowshahi, F., Sarfraz, M., Ursyn, A. (eds.). IEEE Computer Society Press Los Alamitos, (2001) 425-432

22.Craig, P., Kennedy, J.: Concept Relationship Editor: A visual interface to support the assertion of synonymy relationships between taxonomic classifications. Visualization and Data Analysis. SPIE Vol. 6809. SPIE Press Bellingham WA (2008) 12

23.Cockburn, A., Karlson, A., Bederson, B.B.: A Review of Overview+Detail, Zooming, and Focus+Context Interfaces. To appear in ACM Computing Surveys. 41 (2009)

24.Tognazzini, B.: User testing on the cheap TOG on Interface. Addison-Wesley (1992) 79-89

25.Nielsen, J.: Guerrila HCI: Using Discount Usability Engineering to Penetrate the Intimidation Barrier. In: Bias, R.G., Mayhew, D.J. (eds.) Cost-Justifying Usability. Academic Press Professional (1994) 245-272

26.Wixon, D.: Evaluating Usability Methods. Interactions. 10 (2003) 28-34 